\title{
Metastasiertes Kolorektalkarzinom
}

\section{Zulassung für Kombinationstherapien mit Panitumumab}

\begin{abstract}
Die Europäische Kommission hat eine Zulassungserweiterung von Panitumumab genehmigt. Der humane EGFR (Epidermal Growth Factor Receptor)-Antikörper ist in der Behandlung von Patienten mit metastasiertem Kolorektalkarzinom (mCRC) und Wildtyp-KRAS* auch zugelassen als Erstlinientherapie in Kombination mit FOLFOX und als Zweitlinientherapie in Kombination mit FOLFIRI bei Patienten, die in der Erstlinientherapie eine Fluoropyrimidin-haltige Chemotherapie erhalten haben (ausgenommen Irinotecan).
\end{abstract}

Wie in den Phase-III-Studien 20050203 (PRIME) und 20050181 ( 181) gezeigt wurde, kann durch die zusätzliche Gabe von Pani- tumumab (Vectibix ${ }^{\circledast}$ ) zur Chemotherapie mit FOLFIRI (Folinsäure [FS]/5-Fluorouracil [5-FU]//rinotecan) oder FOLFOX (FS/5-FU/ Oxaliplatin) das mediane progressionsfreie Überleben bei Patienten mit mCRC und Wildtyp-KRAS im Vergleich zur alleinigen Chemotherapie verlängert werden (Douillard JE et al., 2010, J Clin Oncol 28: 46974705; Peeters M et al., 2010, J Clin Oncol 28: 4706-4713).

Bei den Patienten mit KRAS-Mutationen waren die Behandlungsergebnisse für die Kombination von Panitumumab mit FOLFOX den Behandlungsergebnissen mit FOLFOX allein unterlegen. Panitumumab darf nur bei Patienten mit nachgewiesenem Wildtyp-KRAS-Status angewendet werden.

\section{Zulassung für die Monotherapie mit Panitumumab überarbeitet}

Panitumumab wurde in der Europäischen Union bereits im Jahr 2007 unter besonderen Bedingungen für die Monotherapie bei Patienten mit mCRC zugelassen. Die bisherige Zulassung für die Monotherapie mit Panitumumab wurde jetzt ebenfalls überarbeitet, um klarzustellen, dass Panitumumab für die Behandlung von Patienten mit mCRC und Wildtyp-KRAS, nach Versagen von Fluoropyrimidin-, Oxaliplatin- und Irinotecan-haltigen Chemotherapieregimen, indiziert ist.

*Kirsten Rat Sarcoma Viral Oncogene Homolog

Quelle: Information der Amgen GmbH

\section{Erhaltungstherapie bei nichtkleinzelligem Lungenkarzinom Kontinuierliche Weiterbehandlung mit Pemetrexed ist zugelassen}

Die Zulassung von Pemetrexed wurde am 26. Oktober 2011 nochmals erweitert: Pemetrexed ist seitdem auch als Mono-Erhaltungstherapie zugelassen bei Patienten mit fortgeschrittenem bzw. metastasiertem nichtkleinzelligem Lungenkarzinom (NSCLC) und Nichtplattenepithelhistologie, deren Erkrankung nach einer platinbasierten Chemotherapie nicht unmittelbar fortgeschritten ist. Die Erhaltungstherapie mit Pemetrexed kann nach Einschätzung von PD Dr. Martin Reck, Großhansdorf, problemlos durchgeführt werden und die Prognose individuell verbessern.

Die Zulassungserweiterung basiert auf den Ergebnissen der PARAMOUNT-Studie (Paz-Ares LG et al., 2011, J Clin Oncol 29 [Suppl]: CRA7510). Diese Phase-III-Studie war laut Reck sehr groß angelegt, um maximale statistische Sicherheit zu liefern: 939 nicht vorbehandelte Patienten mit nichtplattenepithelialem NSCLC (Stadium IIIB, IV) erhielten eine Induktionschemotherapie mit Pemetrexed (Alimta ${ }^{\circledR}$ ) plus Cisplatin für vier Zyklen. In der anschließenden Erhaltungsphase

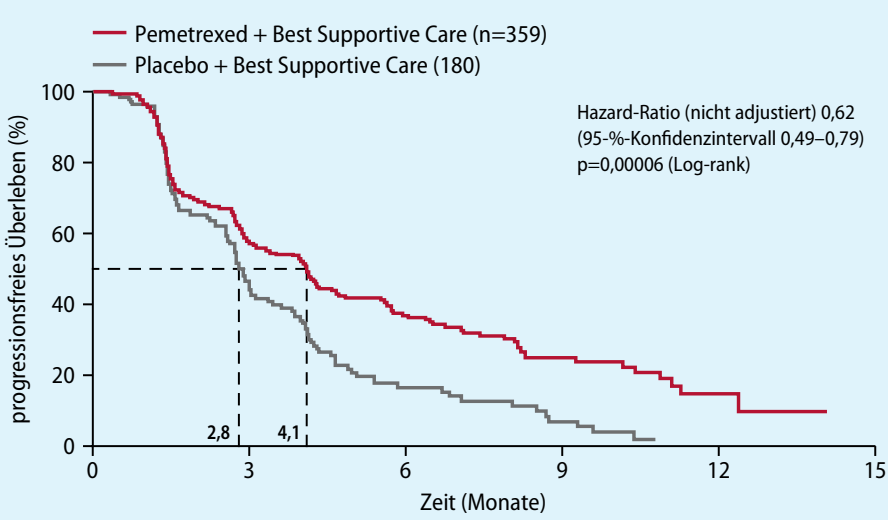

nach paz-Ares LG et al., 2011, J Clin Oncol 29 (Suppl): CRA7510

PARAMOUNT-Studie: Progressionsfreies Überleben ab Randomisierung für die Erhaltungsphase (Prüfarztauswertung; primärer Endpunkt) wurden Patienten, die mindestens eine Tumorstabilisierung erreicht hatten, randomisiert im Verhältnis 2:1 mit Pemetrexed weiterbehandelt oder bekamen Placebo - jeweils bis zur Progression.

\section{Verlängertes Überleben mit Pemetrexed}

In der Beurteilung des primären Endpunkts zeigte sich ein „ganz klares positives Signal", so Reck: Das progressionsfreie Überleben (PFS) ab dem Tag der Randomisierung war in der Prüfarztauswertung unter der sogenannten continuation maintenance mit Pemetrexed signifikant länger als in der Placebogruppe (Abb.). Das PFS ist laut Reck der Parameter, mit dem die Wirksamkeit dieses Therapiesegments am besten beurteilt werden kann. Die ermittelte Hazard-Ratio von 0,62 für die Risikoreduktion bezeichnete Reck als eindrucksvoll verglichen mit den Resultaten vorangegangener Studien bei fortgeschrittenem Lungenkarzinom mit Werten von 0,8 und 0,85. Erfreulich ist laut Reck auch, dass das Ergebnis durch unabhängige Gutachter bestätigt werden konnte (median 3,9 Monate versus 2,6 Monate; Hazard-Ratio 0,64; $p=0,0002$ ). Eine Interimsanalyse zum Gesamtüberleben nach median 11,04 Monaten Nachbeobachtungszeit ergab bereits einen Vorteil zugunsten der Erhaltungstherapie mit Pemetrexed (median 13,9 Monate versus 11,1 Monate; Hazard-Ratio 0,78; $p=0,0034$; Fachinformation Alimta ${ }^{\circledR}$, Oktober 2011). Die Verträglichkeit der Therapie bewertete Reck insgesamt als gut. $A M$

Quelle: Veranstaltung der Lilly Deutschland GmbH 